\title{
FUNDAMENTAL PHYSICS AND RELATIVISTIC LABORATORY ASTROPHYSICS WITH EXTREME POWER LASERS
}

\author{
T.Zh. Esirkepov ${ }^{1}$ and S.V. Bulanov ${ }^{1}$
}

\begin{abstract}
The prospects of using extreme relativistic laser-matter interactions for laboratory astrophysics are discussed. Laser-driven process simulation of matter dynamics at ultra-high energy density is proposed for the studies of astrophysical compact objects and the early universe.
\end{abstract}

\section{Introduction}

The advent of chirped pulse amplification (CPA) has lead to a dramatic increase of lasers' power and intensity (Mourou et al. 2006; Fig. 1). Present-day lasers are capable to produce pulses whose electromagnetic (EM) field intensity at focus is well above $10^{18} \mathrm{~W} / \mathrm{cm}^{2}$. An electron becomes essentially relativistic in such fields, so that its kinetic energy is comparable to its rest energy. Optics in this regime is referred to as relativistic optics; the corresponding EM field is called "relativistically strong". Focused laser intensities as high as $10^{22} \mathrm{~W} / \mathrm{cm}^{2}$ have been reached in experiments (Bahk et al. 2004). The increase of laser intensity opened the field of laser-driven relativistic laboratory astrophysics, where laser-matter interactions exhibit relativistic processes similar to some astrophysical phenomena. Acquiring greater attention from various scientific communities, laboratory astrophysics becomes one of the most important motivations for the construction of the ultra-high power lasers (Bulanov et al. 2008).

In 2010 the CPA laser systems operating worldwide reached the total peak power of 11.5 PW and by the end of 2015 planned CPA facilities, except exawatt scale projects, will bring the total of $127 \mathrm{PW}$ (Barty 2010). The most powerful laser facilities, NIF (LLNL, USA) and LMJ (Bordeaux, France), are intended primarily for inertial thermonuclear fusion. The NIF designed for 1.8 MJ has demonstrated 1.41 MJ with 192 beams of $3 \omega$ in August, 2011. The LMJ will deliver 1.8 MJ

\footnotetext{
1 Kansai Photon Science Institute, Japan Atomic Energy Agency, 8-1-7 Umemi-dai, Kizugawa, Kyoto 619-0215, Japan
} 


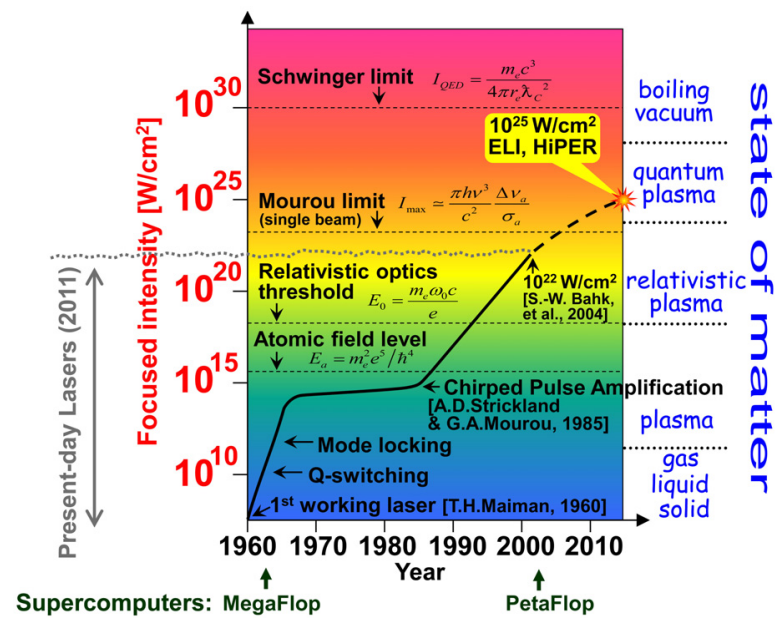

Fig. 1. The progress of laser technology in terms of focused intensity, whose increase enables new states of matter. The investigation of new regimes of laser-matter interactions is bolstered up by the supercomputer performance growth.

with 240 beams in 2012. The PETAL laser coupled with LMJ will produce long pulse beams of $200 \mathrm{~kJ}$ combined with ultra-high intensity beams of $70 \mathrm{~kJ}$ for fast ignition research and fundamental science. Future exawatt scale facilities HiPER (High Power laser Energy Research) and ELI (Extreme Light Infrastructure) are intended for fundamental science, including high field science. ELI, in particular, is designed for producing femtosecond pulses of $70 \mathrm{KJ}$ with power and intensity larger than $100 \mathrm{PW}$ and $10^{25} \mathrm{~W} / \mathrm{cm}^{2}$ respectively. In the plans of all these facilities there are experiments for laboratory astrophysics.

Laboratory astrophysics complements and supports astrophysical observations with laboratory experiments, theoretical and numerical studies, which helps to interpret observations and allows refining the theory by strengthening its predictive power. Laboratory astrophysics applications are categorized into four domains (Savin et al. 2011): atomic and molecular domains, dust and ices domain, and plasma domain. They are focused on the interpretation of observed spectra, identification of astrophysical processes, simulation of some phenomena experimentally in the laboratory and numerically with computers, development of instruments and diagnostics for observational astrophysics. Lasers play important role in all these domains as a tool of diagnostics. In some cases, lasers drive the processes of interest enabling the experimental simulation of astrophysical conditions, e.g. radiatively, magnetically, and kinetically driven laboratory experiments in the plasma domain. Recalling recent results from WMAP and Planck spacecrafts, one can consider the fifth domain, cosmology, where one of intriguing problems is the state of matter in the early universe. Extreme power lasers such as ELI and HiPER can produce conditions close to the lepton epoch of the early universe. 


\section{Laser-matter interaction}

An interaction of an EM wave with matter is principally determined by its intensity (irradiance), $I=c E_{0}^{2} / 4 \pi$, where $E_{0}$ is the electric field amplitude and $c$ is the speed of light in vacuum. For different thresholds of the laser pulse focused intensity different nonlinear effects are produced, Figure 1. (i) At the intensity of $I \simeq 10^{12}-10^{13} \mathrm{~W} / \mathrm{cm}^{2}$ two-photon ionization, above-threshold ionization, and double ionization have been observed using high-order harmonics (corresponding to wavelengths from 30 to $110 \mathrm{~nm}$ ) generated in gases (Sekikawa et al. 2004). (ii) At the intensity of $I_{a}=3.5 \times 10^{16} \mathrm{~W} / \mathrm{cm}^{2}$, which is the atomic unit of intensity corresponding to the atomic unit of electric field $E_{a}=m_{e}^{2} e^{5} / \hbar^{4}$, the interaction of EM pulses with atomic gas is highly nonlinear and is accompanied by harmonic generation (Teubner \& Gibbon 2009). Nonlinearities can start at lower intensities for atoms in the excited states. Here $m_{e}$ and $e$ are the mass and charge of electron, and $\hbar=h / 2 \pi$ is the reduced Planck constant. (iii) The EM wave with wavelength $\lambda$ and frequency $\omega$ causes the electron dynamics to be relativistic at the intensity of $I_{\text {rel }}=1.37 \times 10^{18} \mathrm{~W} / \mathrm{cm}^{2} \times(\mu \mathrm{m} / \lambda)^{2}$, corresponding to the unit value of the dimensionless amplitude of the wave $a_{0}=e E_{0} / m_{e} \omega c$ (Mourou et al. 2006). (iv) At the intensity of $I_{\text {rad }}=2.6 \times 10^{23} \mathrm{~W} / \mathrm{cm}^{2}(\mu \mathrm{m} / \lambda)^{4 / 3}$, the electron dynamics becomes dominated by the radiation reaction (Bulanov et al. 2004). The corresponding dimensionless amplitude is $a=\left(3 \lambda / 4 \pi r_{e}\right)^{1 / 3}$, where $r_{e}=e^{2} / m_{e} c^{2}$ is the classical electron radius. (v) Quantum effects come into play at the intensity of $I_{q u a}=6 \times 10^{24} \mathrm{~W} / \mathrm{cm}^{2}$ (Bulanov et al. 2004). The corresponding electric field is $E_{\text {qua }}=4 \pi e / 3 \lambda_{C}$, where $\lambda_{C}=h / m_{e} c=2.43 \times 10^{-10} \mathrm{~cm}$ is the Compton wavelength of the electron. (vi) At the intensity of $I_{S}=2.3 \times 10^{29} \mathrm{~W} / \mathrm{cm}^{2}$, corresponding to the critical electric field of quantum electrodynamics (QED) or so-called Schwinger field $E_{S}=m_{e}^{2} c^{3} / e \hbar$, electron-positron $\left(e^{-} e^{+}\right)$pairs are created from vacuum (Heisenberg \& Euler 1936; Schwinger 1951) and Unruh radiation (Unruh 1976) of electron in the electric field can become dominant (Chen \& Tajima 1999). Interactions of high-irradiance lasers with matter is described in more details in recent reviews (Mourou et al. 2006; Salamin et al. 2006; Marklund \& Shukla 2006).

The dimensionless amplitude of the laser pulse at focus, $a_{0}$, is related to the laser peak intensity by $I_{0 L}=a_{0}^{2} \times 1.37 \times 10^{18} \mathrm{~W} / \mathrm{cm}^{2} \times(\mu \mathrm{m} / \lambda)^{2}$ for linear and $I_{0 C}=2 I_{0 L}$ for circular polarization. Other important dimensionless parameters characterizing the laser pulse are the number of cycles corresponding to the pulse duration, $\tau_{\text {las }}$, as $N_{\text {las }}=c \tau_{\text {las }} / \lambda$, and the focal spot diameter in terms of laser wavelengths, $D_{\text {las }} / \lambda$. Further, in the interaction of a laser pulse with plasma, a collective response of plasma to a periodic EM wave is characterized by the ratio of the plasma electron number density, $n_{e}$, to critical density, $n_{c r}=m_{e} \omega^{2} / 4 \pi e^{2}$, determined by the laser frequency. It is related to the Langmuir frequency $\omega_{p e}=\left(4 \pi n_{e} e^{2} / m_{e}\right)^{1 / 2}$ via $n_{e} / n_{c r}=\omega_{p e}^{2} / \omega^{2}$. These parameters appear in the dispersion equation of a relativistically strong EM wave with circular polarization propagating in plasma, $\omega^{2}=k^{2} c^{2}+\omega_{p e}^{2}\left(1+a_{0}^{2}\right)^{-1 / 2}$, where $k$ is the wave number. As seen in the experiment, theory and computer simulation, irradiation of 
(a) Observation

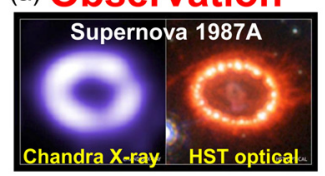

(b) Theory

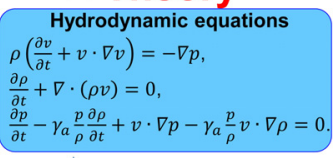

Similarity principles

(d) Experiment (modeling, simulation)

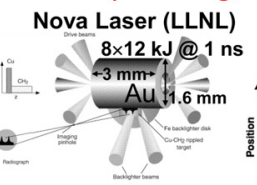

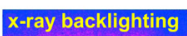

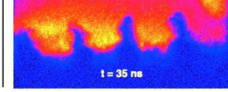

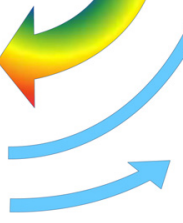

(c) Numerical Simulation

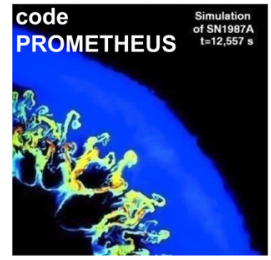

Fig. 2. Process simulation with high-power laser-plasma interactions (d) and configuration simulation with computer models (c) help to refine the theory (b) of observed phenomena (a), as exemplified by the investigation of the supernova shells instabilities (Remington et al. 1999).

various targets - particle bunch, gas, liquid, porous target, solid - by a relativistically strong laser pulse results in a formation of plasmas, laser pulse self-focusing, its frequency and shape modulation, and a generation of strong collective EM fields leading to transformation of the laser pulse energy into the energy of (1) waves of different kinds, such as plasma wake wave and electromagnetic soliton; (2) large-scale electrostatic field and quasi-static magnetic field; (3) EM waves in different frequency range, such as terahertz radiation, high harmonics up to ultraviolet (UV) and eXtreme ultraviolet (XUV), X-ray and $\gamma$-ray; (4) plasma particles (electrons, positive and negative ions); (5) products of nuclear reactions (e.g., neutrons, positrons, muons, etc.). The observation of these new phenomena pointed the way to many promising applications. The corresponding processes can be utilized for creation of useful tools in laboratory setting (such as for diagnostics) and, much beyond specialized scientific needs, in technology.

\section{Similitude}

Using a similarity of scaling laws between the physical system and its model in a laboratory and reproducing the key dimensionless parameters one can study the corresponding physical process in a controllable way, Figure 2. Two systems behave identically if they are described by the same equations with the same values of dimensionless parameters. In astrophysical phenomena modeling, however, such the identity can hardly be achieved because the key dimensional parameters 
(a) Collisionless shock waves in space
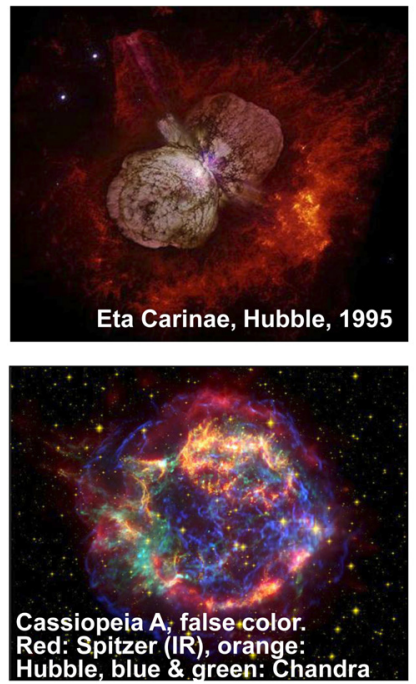

(b) Laser-driven collisionless shock waves

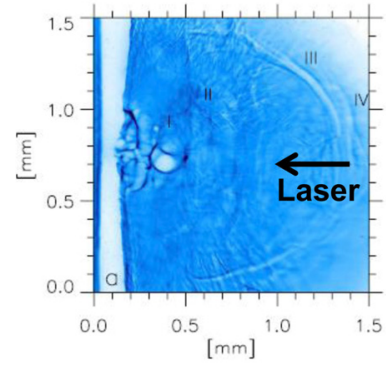

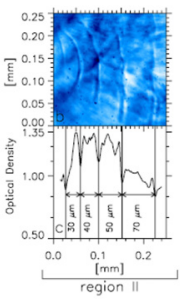
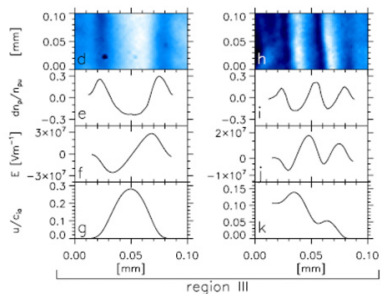

Fig. 3. Collisionless shock waves seen in space (a). In laser plasma (b) the shock wave motion is detected with proton imaging and studied with computer modeling (Romagnani et al. 2008).

(a) Wake waves in space
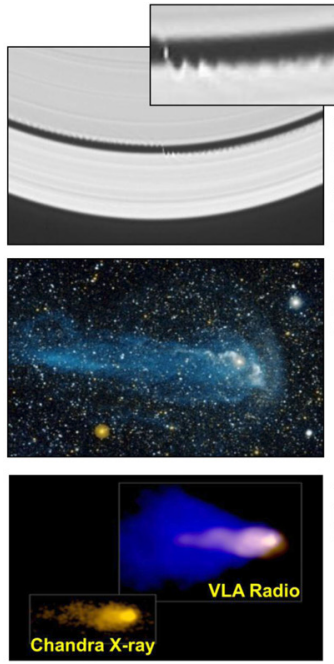

Wake behind a small moon in the

Keeler gap in

Saturn's rings.

Cassini spacecraft.

Tail behind the

Mira star.

GALEX satellite.

(d) Ship wake

Tail behind the Mouse pulsar G359.23-0.82

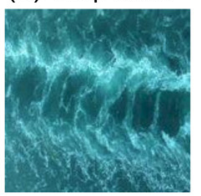

\section{Laser-driven wake waves}

(b)

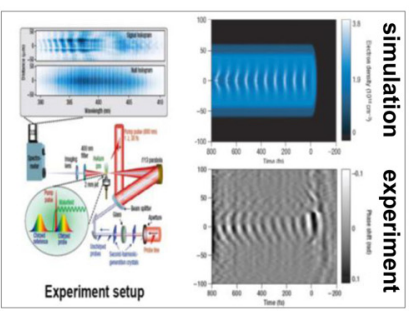

(C) Ti:Sa Salle Jaune laser 2J@35fs
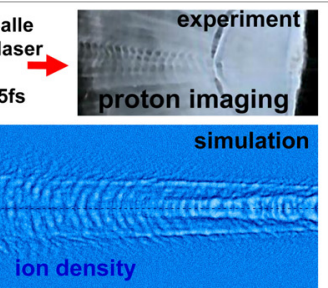

Fig. 4. Wake waves seen in space (a) and ocean (d). In laser plasma wake wave manifests itself by a fast change of electron density (b) snap-shot by frequency-domain holography (Matlis et al. 2006) and relatively slow response of ions (c) imaged with MeV protons (Borghesi et al. 2005). 
appear to be prohibitively large or small and because an analytical theory of astrophysical phenomena is often far from being complete. Fortunately, an approximate (incomplete) similarity with only few basic parameters is helpful for the development and refinement of the theory. A so-called configuration simulation tries to reconstruct the global geometry and some physical processes therein, while a process simulation reproduces local properties of physical processes at astrophysical conditions (Fälthammar 1974). An important case of the approximate similarity is limited scaling or limited similarity, where it is sufficient that "dimensionless quantities in nature which are small compared to unity should be small in the model, but not necessarily by the same order of magnitude" (Block 1967). Laser-matter interactions provide many examples of similarity with astrophysical phenomena and allows an insight into fundamental processes at astrophysical conditions.

Shocks developed during a supernova explosion and Rayleigh-Taylor and Richtmayer-Meshkov instabilities of the supernova shells create large scale modulations of the gas density. The same type of instabilities are typically developed in the interaction of high-power lasers with dense materials. Laboratory experiments with laser-driven shocks in high density plasma reveal fundamental properties of materials such as opacities and equations of state, and allow refining of numerical simulations and analytical models for supernovae shocks development and evolution (Remington et al. 1999; Fig. 2). Underlying one of the mechanisms of cosmic ray acceleration, shock waves propagate in interstellar plasma. On later stages of evolution, when their amplitude is relatively small, they can be described by Korteweg-de Veries-Burgers equation. In high intensity laser-plasma experiments, Figure 3, the collisionless shock structure and corresponding electric field have been studied with high spatial and temporal resolution owing to proton imaging technique (Romagnani et al. 2008).

Relative motion of compact objects and interstellar media produce various wakes and (shock) bow waves. In laboratory plasma these structures can be simulated to some extent by a relativistically strong laser pulse creating wake waves (Matlis et al. 2006; Borghesi et al. 2005; Fig. 4) and bow waves (Esirkepov et al. 2008, Fig. 5) in underdense plasma.

The magnetic field lines reconnection visible in solar flares leads to a fast magnetic energy release into motion and heating of plasma, EM radiation emission and charged particle acceleration. Magnetic reconnection, Figure 6, can be studied in the laboratory using high-power lasers (Nilson et al. 2006; Zhong et al. 2010).

Radiation pressure plays important role in astrophysical environments. High intensity ultrashort laser pulses irradiating thin foils can produce radiation pressure which dominates the plasma dynamics, leading to efficient collective acceleration of ions to relativistic energy (Bulanov et al. 2004; Esirkepov et al. 2004; Kar et al. 2008; Fig. 7).

Relativistically rotating compact objects emitting periodic pulses of high-frequency radiation are exemplified in astrophysics by pulsars. In laser plasma a relativistic rotator can be modeled with a circularly polarized electromagnetic quasi-soliton (Esirkepov et al. 2004; Fig. 8). 
(a) Bow waves in space
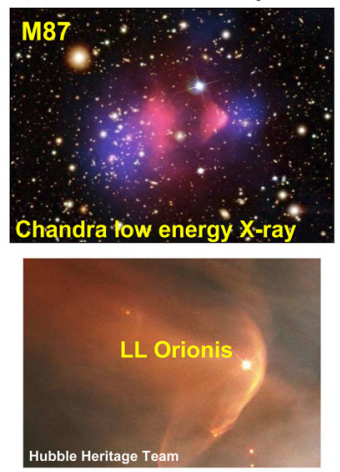

(b) Laser-driven bow wave

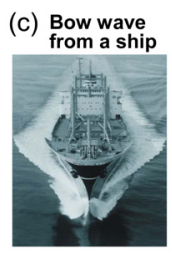
PIC simulation
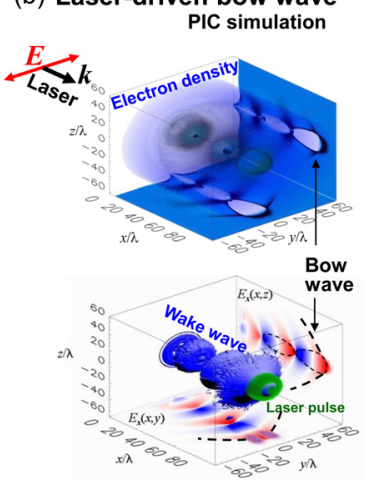

Fig. 5. Bow wave induced in ambient medium by a compact object as seen in space (a), ocean (c) and laser plasma simulations (b). In plasma the role of the compact object is played by a tightly focused laser pulse (Esirkepov et al. 2008).

\section{Reaching astrophysical conditions with extreme power lasers}

Extreme EM fields and ultra-high energy and matter densities are present in various astrophysical objects, as follows from the observation of ultra-high-energy cosmic rays (Abraham et al. 2008), gamma ray bursts (Vedrenne \& Atteia 2009), radiation from pulsars (Lorimer \& Kramer 2004), etc. Extreme power lasers such as HiPER and ELI enable the process simulation of matter dynamics at ultra-high energy density in astrophysical compact objects and in the early universe. With the laser-driven charged particle acceleration, present-day lasers are capable of producing physical conditions which where accessible before in laboratory only in high energy physics in the regime of a few particles interaction. Combining multiple high-power laser beams into a tight focus (Bulanov et al. 2010) or using relativistic mirrors formed by plasma wake waves (Bulanov et al. 2003), one can build up the critical field of quantum electrodynamics (Schwinger field) in a "macroscopic" volume (with the scale length much greater than the Compton wavelength of the electron). This gives a tool for studying collective effects of quantum electrodynamics.

\subsection{Towards Schwinger field with relativistic flying mirror}

The change of EM radiation frequency emitted by or reflected from moving objects (Doppler effect) is ubiquitous in astrophysical observations. In laser plasma this effect can be used for the intensification of EM radiation.

A relativistically strong laser pulse (driver) propagating in low-density plasma efficiently creates a wake wave (Akhiezer \& Polovin 1956; Tajima \& Dawson 1979) when the pulse duration is much less than the inverse Langmuir frequency of plasma, $\omega_{p e}^{-1}$, Figure 9 . The wake wave phase velocity $v_{p h}=\beta_{p h} c$ equals the 
(a) Magnetic line reconnection in space
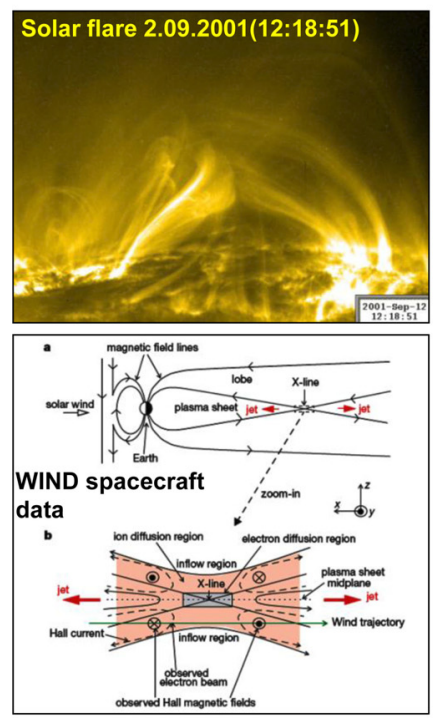

\section{Laser-driven magnetic line reconnection (experiment)}

(b)

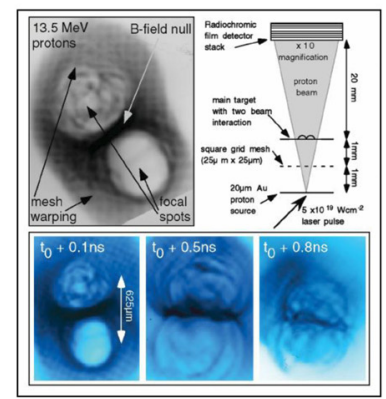

(c)

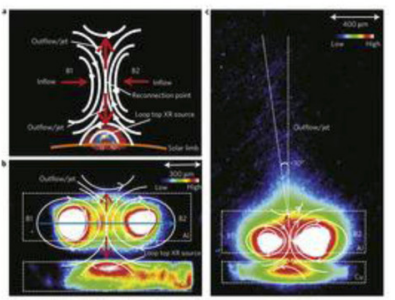

Fig. 6. The reconnection of magnetic field lines seen in solar flares (a) and in high-power laser-plasma interactions ((b) Nilson et al. 2006; (c) Zhong et al. 2010).

(a) Radiation pressure in space
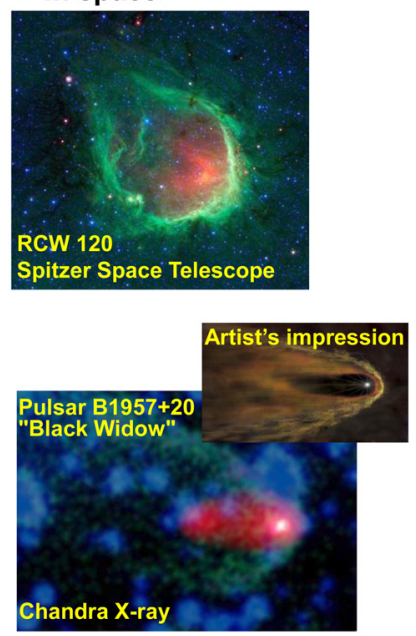

(b) Laser radiation pressure

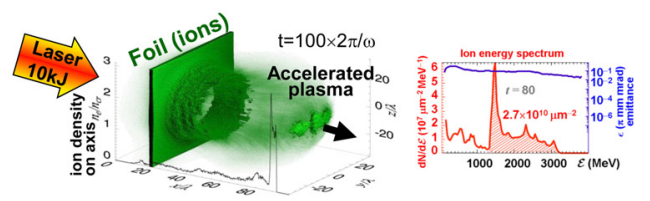

(c) VULCAN Nd-glass laser (RAL) 60 J@1ps \& $250 \mathrm{~J} @ 0.7 \mathrm{ps}$; foils $(3,5$ mum, Al \& Cu)

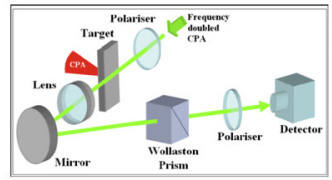

experiment
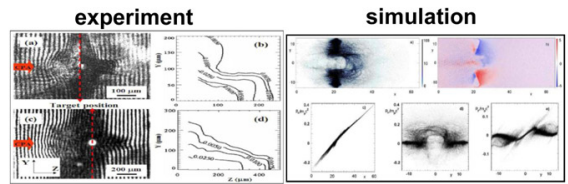

Fig. 7. Electromagnetic radiation pressure revealed in space (a) and in laser plasma with (b) computer simulation (Bulanov et al. 2004; Esirkepov et al. 2004) and in (c) experiments (Kar et al. 2008). 
(a) Relativistic rotator in space
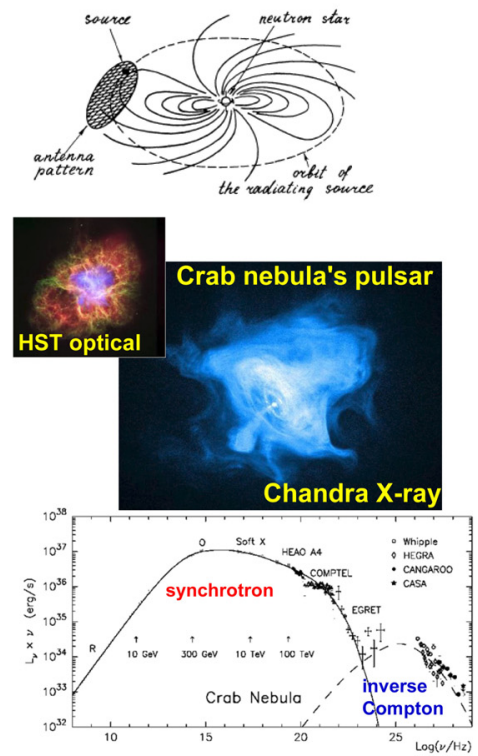

(b) Laser-driven relativistic rotator (simulation)
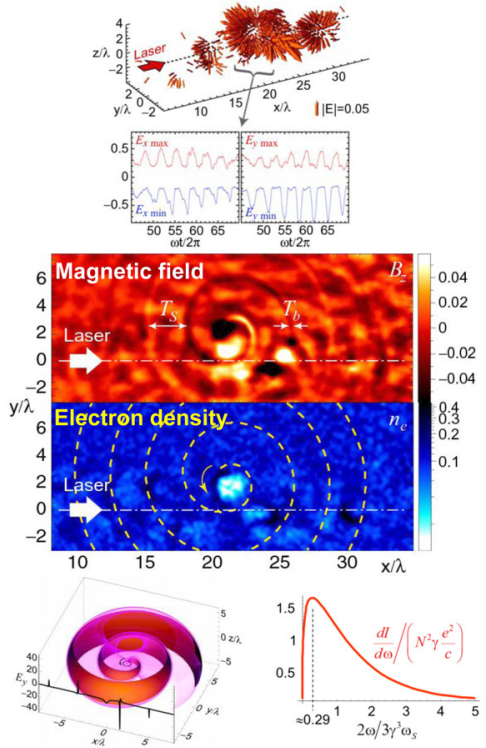

Fig. 8. Relativistic rotators seen due to their emission in space (a) and in laser plasma simulation (b), where the emitter is a circularly polarized electromagnetic quasi-soliton (Esirkepov et al. 2004).

laser pulse group velocity which is close to the speed of light in vacuum. The corresponding Lorentz factor is $\gamma_{p h}=\left(1-\beta_{p h}^{2}\right)^{-1 / 2} \approx \omega / \omega_{p e}$, where $\omega$ is the laser pulse frequency. When the laser dimensionless amplitude is sufficiently large, $a_{0} \geq\left(4 n_{c r} / n_{e}\right)^{1 / 3}$ (Zhidkov 2004), the wake wave is breaking. Near breaking the electron density profile in the wake takes the shape of spikes separated by the wake wavelength, $\lambda_{p} \approx 4\left(2 \gamma_{p h}\right)^{1 / 2} c / \omega_{p e}$ for $\gamma_{p h} \gg 1$. Due to the dependence of the wake wave frequency on the driver pulse amplitude, in the transverse direction the spikes continue as thin approximately paraboloidal shells (Bulanov \& Sakharov 1991), Figure 9. These shells can partially reflect and focus a counter-propagating laser pulse (source). Due to a strong localization of the electron density in the shells, for sufficiently long EM waves the geometric optics approximation fails and the reflection coefficient is not exponentially small. The reflected wave vectorpotential scales as $\gamma_{p h}^{-3}$ while the reflected energy flux grows as $\gamma_{p h}$ due to a large frequency upshift, $\omega_{r} \approx 4 \gamma_{p h}^{4} \omega$. In addition, due to focusing the reflected wave intensity is increased by the factor of $8(D / \lambda)^{2} \gamma_{p h}^{3}$ (at focus) with respect to the incident wave, where $D$ is the diameter of the reflecting shell. This favorable scaling allows reaching Schwinger field at which $e^{-} e^{+}$pairs are created from vacuum with present-day laser technology (Bulanov et al. 2003). The frequency upshift of the laser pulse reflected from relativistic flying mirrors formed by the plasma wake wave has been observed in proof-of-principle experiments (Kando et al. 2007). 

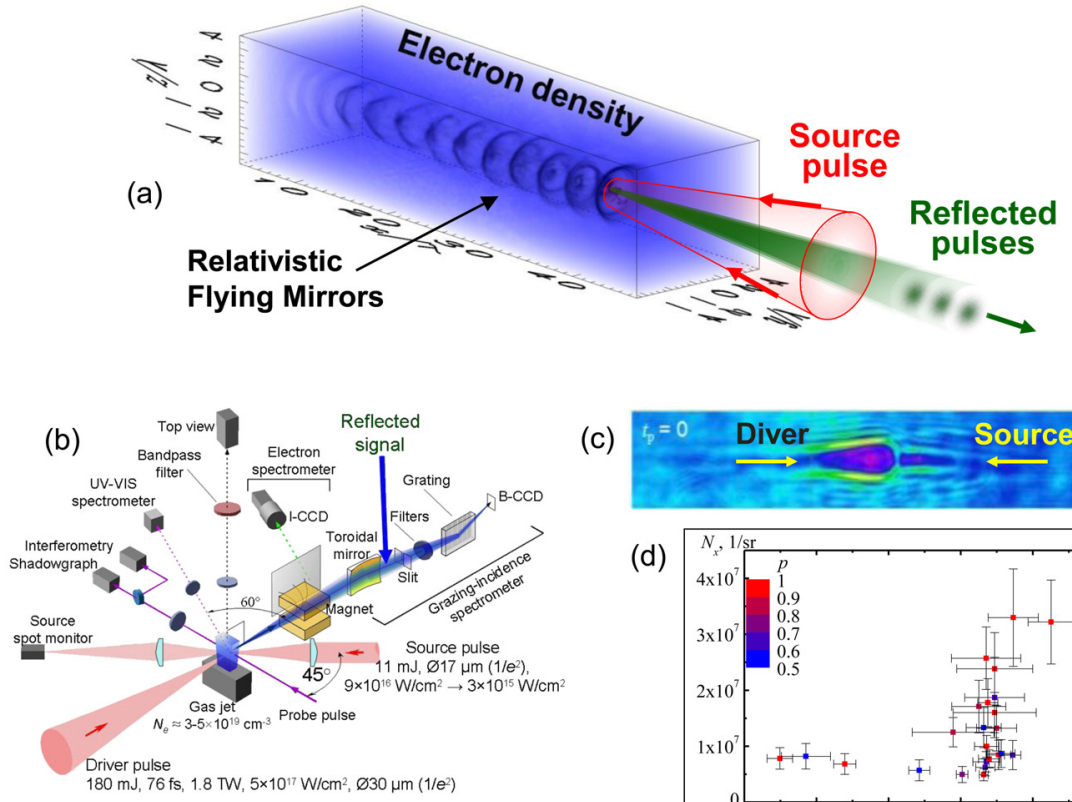

(c)

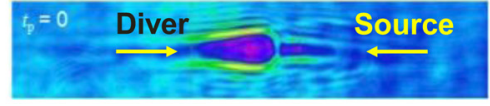

(d)

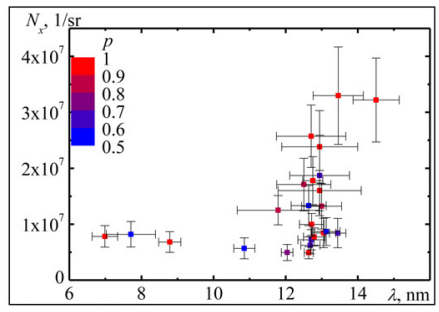

Fig. 9. The relativistic flying mirrors formed by electron density modulations in the wake wave (a). A counter-propagating laser beam is partially reflected with a large frequency upshift. The proof-of-principle experiment setup (b) colliding two pulses (c) signal (d).

The curvature of the relativistic flying mirror is controlled by the transverse profile of the driver pulse. The EM wave in the beam reflected from a flat flying mirror is almost planar and can not create $e^{-} e^{+}$pairs, since both the fundamental invariants of the EM field, $\mathbf{E}^{2}-\mathbf{B}^{2}$ and $\mathbf{E} \cdot \mathbf{B}$, are zero. In the case of ideally parabolic flying mirror, the reflected beam focuses into a moving spot and then diverges within the collimation angle of $\sim 1 / \gamma_{p h}$. The first fundamental invariant is of the order of $\mathbf{E}^{2} / \gamma_{p h}^{2}$, so that the magnitude of the reflected beam can exceed Schwinger field by the factor of $\gamma_{p h}$ without making vacuum unstable. Varying an angle of the collision of two such beams one can study different regimes of the $e^{-} e^{+}$pairs creation from vacuum.

\subsection{Laser-driven electron-positron-gamma plasma}

Astrophysical observations of EM radiation from compact objects such as pulsars and active galactic nuclei reveal the important role of the processes of $e^{-} e^{+}$pairs creation from vacuum in strong EM fields and abundant gamma-ray generation. Understanding the mechanisms of vacuum breakdown and polarization is challenging for nonlinear quantum field theories and for astrophysics (Ruffini et al. 2010). These fundamental processes occurring in neutron star magnetospheres, sources of gamma ray bursts, and during the lepton epoch of the early universe, can be 

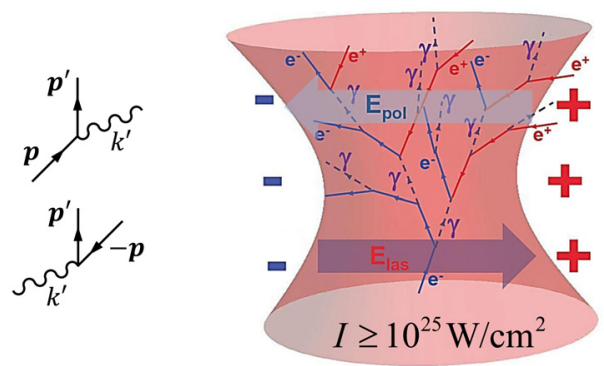

Fig. 10. Electron-positron-gamma plasma created at the focus of extreme-power laser.

produced in a terrestrial laboratory with extreme power lasers using present day technology.

As mentioned in Section 2, the characteristic value of the electric field in which vacuum becomes unstable is $E_{S}=m_{e}^{2} c^{3} / e \hbar=1.3 \times 10^{16} \mathrm{~V} / \mathrm{cm}$ corresponding to the intensity of $I_{S}=2.3 \times 10^{29} \mathrm{~W} / \mathrm{cm}^{2}$. However, at a laser focus with a few micron diameter (much larger than the Compton wavelength of the electron) the vacuum becomes unstable and $e^{-} e^{+}$pairs are created at two-three orders of magnitude less intensity, well before the laser field reaches the Schwinger limit, due to a large phase volume occupied by a high-intensity EM field (Bulanov et al. 2006), Figure 10.

If an electron (seed) get into a laser focus at the intensity of the order of $10^{25} \mathrm{~W} / \mathrm{cm}^{2}$, it emits gamma photons due to acceleration by the laser EM field in the regime of a strong radiation friction. Some gamma-photons emitted interact with photons comprising the laser field and produce $e^{-} e^{+}$pairs via multi-photon Breit-Wheeler process (Breit \& Wheeler 1934). Then the pairs created generate another portion of gamma-photons, powering an avalanche where electron-positrongamma plasma $\left(e^{-} e^{+} \gamma\right.$ ) is created (Bell \& Kirk 2008; Fedotov et al. 2010; Bulanov et al. 2010). The intensity threshold for the avalanche strongly depends on the laser field polarization, being several orders of magnitude higher in the case of linear polarization, which thus allow an observation of $e^{-} e^{+}$pair creation from vacuum without seed electrons and avalanche (Bulanov et al. 2010).

In addition to the dimensionless amplitude of the electric field, in QED new key parameters emerge in the description of the charged particle interaction with EM fields. The parameter characterizing the probability of the photon emission by the electron moving with 4-momentum $p$ in the the EM field given by the tensor $F^{\mu \nu}$, corresponding to electric field strength $E$, is $\chi_{e}=e \hbar\left|F^{\mu \nu} p_{\nu}\right| / m_{e}^{3} c^{4} \approx$ $\left(E / E_{S}\right)\left(2 p_{\perp} / m_{e} c\right)$ Here $p_{\perp}$ is the electron momentum component perpendicular to the electric field. In the electron rest frame of reference, $\chi_{e} \sim E / E_{S}$. Another parameter characterizes the probability of the $e^{-} e^{+}$pair creation due to the interaction of the EM field with a high energy photon with 4-momentum $\hbar k$ and energy $\omega_{\gamma}: \quad \chi_{\gamma}=e \hbar^{2}\left|F^{\mu \nu} k_{\nu}\right| / m_{e}^{3} c^{4} \approx\left(E / E_{S}\right)\left(2 \hbar \omega_{\gamma} / m_{e} c^{2}\right)$. The probability of the avalanche and $e^{-} e^{+} \gamma$ plasma generation via the multi-photon Breit-Wheeler process is high when these two parameters are close to unity. 


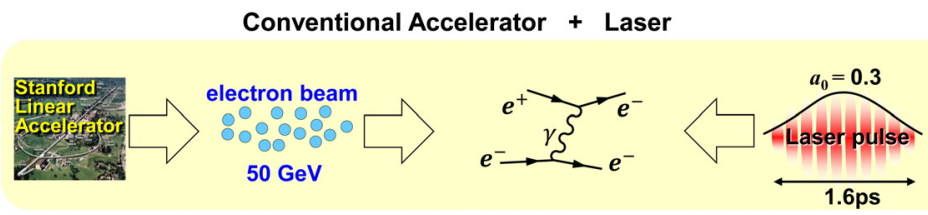

Fig. 11. The experiment on the interaction of intense laser pulse with electron bunch accelerated at the SLAC (Bula et al. 1996). Electron-positron pairs were created via multiphoton inverse Compton scattering and multiphoton Breit-Wheeler process.

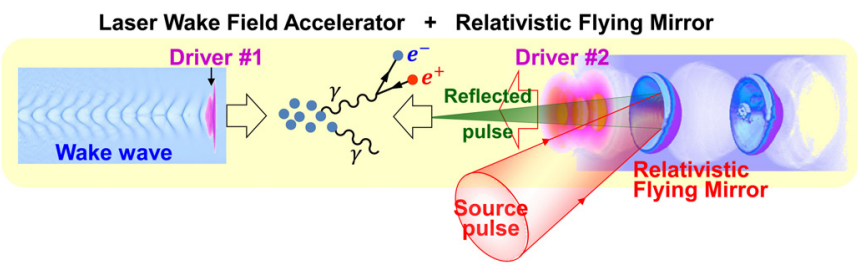

Fig. 12. Proposed experiment on the electron-positron-gamma plasma creation. Laser wake field accelerator created by a laser pulse (driver 1) provides high-energy electron bunches that collide with an ultra-short super-intense electromagnetic pulse from the relativistic flying mirror created by two other laser pulses (driver 2 and source).

The requirements on the laser intensity securing $e^{-} e^{+} \gamma$ plasma creation can be substantially relaxed in the interaction of the laser pulse with a counterpropagating electron beam with sufficiently high energy. In the experiments on the $527 \mathrm{~nm}$ terawatt laser interaction with $46.6 \mathrm{GeV}$ electrons from the SLAC accelerator beam, positron generation was observed (Bula et al. 1996), Figure 11. The laser photons were scattered by the electrons that generated high-energy photons that collided with other laser photons to produce an electron-positron pair. These two steps correspond to multiphoton inverse Compton scattering and multiphoton Breit-Wheeler process, respectively. In the reference frame of electrons the electric field magnitude of the incident radiation was approximately $25 \%$ of Schwinger field. The values of the key parameters introduced above were $\chi_{e} \approx 0.3$ and $\chi_{\gamma} \approx 0.15$.

Recent advances in the developing of the laser wake field accelerator (LWFA) of electrons (Tajima \& Dawson 1979; Leemans et al. 2006; Hafz et al. 2008) enabled an all-optical approach (Bulanov et al. 2011), Figure 12. A sub-picosecond electron bunch with the energy of a few $\mathrm{GeV}$ is accelerated in the wake wave generated by a petawatt laser pulse in plasma. Electrons are injected from plasma into the accelerating phase of the wake wave due to wave-breaking. The accelerated electron bunch collide with an extremely intense EM pulse generated by the flying mirror, produced by two other laser pulses. (In principle, the driver pulse for the LWFA can be the source for the flying mirror). The setup can be optimally synchronized with femtosecond accuracy. As in the case of the experiment at SLAC, the collision between the electron bunch from the LWFA and the EM pulse 


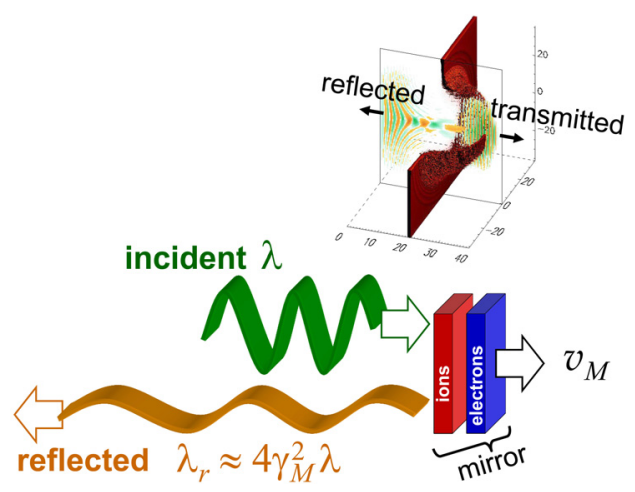

Fig. 13. Radiation pressure dominant acceleration of ions. On the top: the laser pulse is reflected with a gradual decrease of wavelength while ions get accelerated as seen in numerical simulations (Esirkepov et al. 2004).

from the flying mirror produces gamma-photons via Thomson or inverse Compton scattering and then the gamma-photons interact with the laser field generating $e^{-} e^{+}$pairs. For $1.25 \mathrm{GeV}$ electrons, the source pulse amplitude, focal spot size and duration of $a_{s r c}=1, D_{s r c}=10 \mu \mathrm{m}$ and $\tau_{s r c}=30 \mathrm{fs}$, respectively, and the flying mirror Lorentz factor of $\gamma_{F M}=5$, we obtain that the EM field strength at the focus of the flying mirror, $E_{F M}$, increases with respect to the field strength in the source pulse, $E_{s r c}$, as $E_{F M}=2^{5 / 2} \gamma_{F M}^{3 / 2}\left(D_{s r c} / \lambda_{0}\right) E_{s r c} \approx 1.5 \times 10^{-3} E_{S}$, and the key parameters are $\chi_{e} \approx 2 \gamma_{e} E_{F M} / E_{S}=7.5$ and $\chi_{\gamma} \approx\left(2 \hbar \omega_{\gamma} / m_{e} c^{2}\right)\left(E_{F M} / E_{S}\right) \approx$ $1.2 \gamma_{e} E_{F M} / E_{S}=4.5$.

\subsection{Laser-driven collider for quark-gluon plasma studies}

EM radiation pressure is important in astrophysical environments, e.g. it is dominating in formation of nebula surrounding $\eta$ Carinae (van Boekel R. et al. 2003). Multi-petawatt femtosecond laser pulses exert radiation pressure which can dominate in the interaction with matter (Bulanov et al. 2004) realizing one of the most efficient mechanisms of ion acceleration (Esirkepov et al. 2004). This radiation pressure dominant acceleration (RPDA) produces high quality ion bunches with relativistic energy and high luminosity, providing physical conditions for quarkgluon plasma generation (Ludlam \& McLerran 2003).

The RPDA is realized when an extreme power laser pulse pushes forward an irradiated region of a thin foil. Plasma is accelerated as a whole, but ions acquire almost all the energy because of a large ion-to-electron mass ratio. The accelerated foil soon become relativistic and acts as a receding mirror, reflecting the laser pulse, Figure 13. Since the reflected wave frequency decreases by the factor of $4 \gamma_{M}^{2}$, where $\gamma_{M}$ is the mirror Lorentz factor, the laser energy is almost completely transformed into the energy of fast ions. The accelerated foil motion is described by the equation $d / d t\left\{p_{i}+2\left[p_{i}^{3}+\left(m_{i}^{2} c^{2}+p_{i}^{2}\right)^{3 / 2}\right] / 3 m_{i}^{2} c^{2}\right\}=E_{0}^{2} / 2 \pi n_{e} l$, where $p_{i}$ and $m_{i}$ are the ion momentum and mass, $n_{e}$ and $l$ are the electron density and 


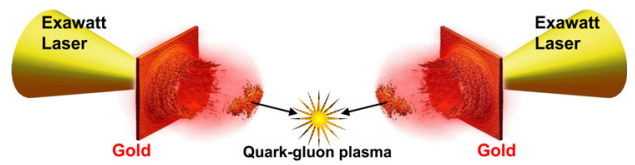

Fig. 14. Laser-driven collider for quark-gluon plasma studies.

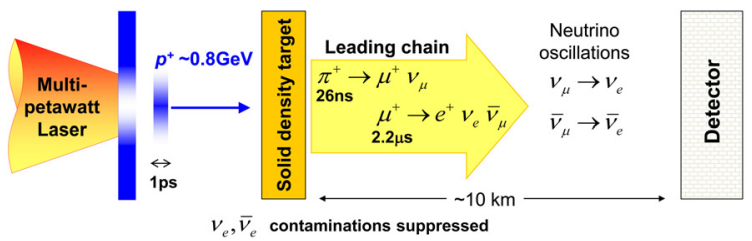

Fig. 15. Laser-driven beam dump facility for neutrino oscillations studies.

thickness of the foil, respectively. By analogy with the self-consistent motion of the electron undergoing Thomson scattering in an EM wave (Landau \& Lifshitz 1951), while in our case the role of the Thomson cross section is played by the quantity $2 / n_{e} l$, the asymptotic energy gain is $\mathcal{E}_{i} \approx m_{i} c^{2}\left(e E_{0}^{2} t / 8 \pi n_{e} l m_{i} c\right)^{1 / 3}$. The laser-to-ion energy transformation efficiency is $\mathcal{N}_{i} \mathcal{E}_{i} / \mathcal{E}_{L}=2 \mathcal{E}_{L} /\left(2 \mathcal{E}_{L}+\mathcal{N}_{i} m_{i} c^{2}\right)$, where $\mathcal{E}_{L}$ is the laser pulse energy and $\mathcal{N}_{i}$ is the ion number. If the laser pulse is sufficiently long, almost all its energy is transformed into ions.

A collision of two heavy ion beams accelerated up to the energy over $100 \mathrm{GeV}$ per nucleon by exawatt laser pulses, Figure 14, can provide the same number of events of quark-gluon plasma generation in one shot as the Relativistic Heavy Ion Collider (RHIC) in few months.

\subsection{Laser-driven beam dump facility for neutrino oscillations studies}

The radiation pressure dominant acceleration of protons in the $\mathrm{GeV}$ energy range can give a source of low-energy (10-200 MeV) neutrinos, which is suitable for neutrino oscillation studies at the "atmospheric" scale (Bulanov et al. 2005). In such the source a high-current beam of protons is stopped by a solid density target, producing pions, Figure 15, in analogy with the LAMPF (presently LANSCE) beam dump facility supplied neutrinos for the LSND experiment (Athanassopoulos et al. LSND). Neutrinos are created with energy of the order of a few tens of $\mathrm{MeV}$ through $\pi^{+} \rightarrow \mu^{+} \nu_{\mu}$ decay-at-rest (DAR) chain and through the subsequent $\mu^{+} \rightarrow e^{+} \nu_{e} \tilde{\nu}_{\mu}$ decay, while the intrinsic $\nu_{e}\left(\tilde{\nu}_{e}\right)$ beam contamination can be kept below 0.1 below the kaon and heavier mesons production threshold $(<3 \mathrm{GeV})$. The oscillations $\nu_{\mu} \rightarrow \nu_{e}$ and $\tilde{\nu}_{\mu} \rightarrow \tilde{\nu}_{e}$ can be simultaneously observed at baselines of $L \sim 10 \mathrm{~km}$ (Bulanov et al. 2005). Measuring the probabilities of these oscillations at the "atmospheric" scale one can refine the constrain on the angle $\theta_{13}$ appearing in the Pontecorvo-Maki-Nakagawa-Sakata (PMNS) matrix, which characterizes mixing between different types of neutrino. Such measurement is the most challenging task for neutrino oscillation experiments. If $\theta_{13}$ is significantly smaller than the current limit $\left(\lesssim 10^{\circ}\right)$, traditional accelerating techniques will be 
unable to provide the required intensity and purity. The laser-driven facility could allow the study of subdominant $\nu_{\mu} \rightarrow \nu_{e}$ oscillations at the "atmospheric" scale for the first time. In addition, such a facility can be developed in conjunction with projects for inertial confined nuclear fusion and neutron spallation sources (Terranova et al. 2006).

\section{Conclusion}

Extreme-power lasers such as ELI and HiPER will allow process simulation for relativistic astrophysics and will lead to new discoveries of collective phenomena in physics. The state of matter peculiar to cosmic gamma ray bursts, the lepton epoch and hadron epoch of the early universe, and processes, which were accessible on Earth only in the high energy physics experiments with conventional accelerators of charged particles, can be produced with the help of super-intense lasers. Thus the lasers entering the realm of extreme intensities become tools for driving and studying processes in the cosmology domain of laboratory astrophysics.

\section{References}

Abraham, J., et al., 2008, Astropart. Phys., 29, 188

Akhiezer, A.I., \& Polovin, R.V., 1956, Sov. Phys. JETP, 30, 915

Athanassopoulos, C., et al., 1997, Nucl. Instr. Meth. A, 388, 149

Bahk, S.-W., et al., 2004, Opt. Lett., 29, 2837

Barty, C.P.J., ICUIL, http://www.icuil.org/

Bell, A.R., \& Kirk, J.G., 2008, Phys. Rev. Lett., 101, 200403

Block, L.P., 1967, Planet. Space Sci., 15, 1479

Boekel, van, R., et al., 2003, A\&A, 410, L37

Borghesi, M., et al., 2005, Phys. Rev. Lett., 94, 195003

Breit, G., \& Wheeler, J.A., 1934, Phys. Rev., 46, 1087

Bula, C., et al., 1996, Phys. Rev. Lett., 76, 3116

Bulanov, S.V., \& Sakharov, A.S., 1991, JETP Lett., 54, 203

Bulanov, S.V., et al., 2003, Phys. Rev. Lett., 91, 085001

Bulanov, S.V., et al., 2004, Plas. Phys. Reports, 30, 196

Bulanov, S.V., et al., 2005, Nucl. Instrum. Meth. A, 540, 25

Bulanov, S.V., et al., 2009, Eur. Phys. J. D, 55, 483

Bulanov, S.V., et al., 2011, Nucl. Instr. Meth. A, 660, 31

Bulanov, S.S., et al., 2006, JETP, 102, 9

Bulanov, S.S., et al., 2010, Phys. Rev. Lett., 104, 220404

Bulanov, S.S., et al., 2010, Phys. Rev. Lett., 105, 220407

Chen, P., \& Tajima, T., 1999, Phys. Rev. Lett., 83, 256

ELI, Extreme Light Infrastructure, http://www.extreme-light-infrastructure.eu/

Esirkepov, T., et al., 2004, Phys. Rev. Lett., 92, 175003

Esirkepov, T., et al., 2004, Phys. Rev. Lett., 92, 255001 
Esirkepov, T., et al., 2008, Phys. Rev. Lett., 101, 265001

Fälthammar, C.-G., 1974, Space Sci. Rev., 15, 803

Fedotov, A.M., et al., 2010, Phys. Rev. Lett., 105, 080402

Hafz, N.A.M., et al., 2008, Nature Photonics, 2, 571

Heisenberg, W., \& Euler, H.Z., 1936, Z. Phys., 98, 714

HiPER, High Power laser Energy Research, http://www.hiper-laser.org/

Kando, M., et al., 2007, Phys. Rev. Lett., 99, 135001

Kar, S., et al., 2008, Phys. Rev. Lett., 100, 225004

Landau, L.D., \& Lifshitz, E.M., 1951, "The Classical Theory of Field" (Addison-Wesley

Press, U. of Michigan, 2nd ed.), chapter 9, problem 6, p. 236

LANSCE, Los Alamos Neutron Science Center, http://lansce.lanl.gov/

Leemans, W.P., et al., 2006, Nature Phys., 2, 696

LMJ, Laser MégaJoule, http://www-lmj.cea.fr/

Lorimer, D.R., \& Kramer, M., 2004, "Handbook of Pulsar Astronomy" (Cambridge University Press)

Ludlam, T., \& McLerran, L., 2003, Phys. Today, 56, 48

Marklund, M., \& Shukla, P.K., 2006, Rev. Mod. Phys., 78, 591

Matlis, N.H., et al., 2006, Nature Phys., 2, 749

Mourou, G.A., et al., 2006, Rev. Mod. Phys., 78, 309

NIF, National Ignition Facility, https://lasers.llnl.gov/

Nilson, P.M., et al., 2006, Phys. Rev. Lett., 97, 255001

PETAL, Petawatt Aquitaine Laser, http://petal.aquitaine.fr/

Planck space observatory, http://www.esa.int/SPECIALS/Planck/index.html

Remington, B.A., et al., 1999, Science, 284, 1488

RHIC, Relativistic Heavy Ion Collider, http://www.bnl.gov/rhic/

Romagnani, L., et al., 2008, Phys. Rev. Lett., 101, 025004

Ruffini, R., et al., 2010, Phys. Rep., 487, 1

Salamin, Y.I., et al., 2006, Phys. Reports, 427, 41

Savin, D.W., 2011 [astro-ph.IM] [arXiv: 1103.1341v1]

Schwinger, J., 1951, Phys. Rev., 82, 664

Sekikawa, T., et al., 2004, Nature, 432, 605

Tajima, T., \& Dawson, J.M., 1979, Phys. Rev. Lett., 43, 267

Terranova, F., et al., 2006, Nucl. Instrum. Meth. A, 558, 430

Teubner, U., \& Gibbon, P., 2009, Rev. Mod. Phys., 81, 445

Unruh, W., 1976, Phys. Rev. D, 14, 870

Vedrenne, G., \& Atteia, J.-L., 2009, "Gamma-ray bursts: the brightest explosions in the universe" (Springer)

WMAP, Wilkinson Microwave Anisotropy Probe, http://map.gsfc.nasa.gov/

Zhidkov, A., et al., 2004, Phys. Rev. E, 69, 035401(R)

Zhong, J., et al., 2010, Nature Phys., 6, 984 